\title{
3D-Fernsehen - Top oder Flop? Eine Marktanalyse
}

\author{
Jenny Baumann, Sarah Bruhs, Yelena Bryantseva, Ekaterina Chmyreva, \\ Marie-Christin Ernst, Anne Frischbier, Katharina Fritsch, Mario Glowik, \\ Lisa Lippert, Bo Ma, Thuy Nguyen und Nathalie Sroka
}

Zusammenfassung

Nach Einschätzungen mehrerer Firmen aus der Unterhaltungselektronik während der »Internationalen Funkausstellung« (IFA) in Berlin 2010 und 2011 erwartet die Branche für die Zukunft vielversprechende Wachstumsraten für Fernsehgeräte mit integrierter 3D-Technologie. Diese relativ optimistische Prognose wurde zum Anlass genommen, eine Marktstudie mit dem Ziel durchzuführen, das Marktpotenzial für Fernsehgeräte mit 3D-Technologie zu untersuchen. Insgesamt wurden 261 Personen aus dem Raum Berlin-Brandenburg befragt, um Rückschlüsse auf deren Kaufbereitschaft für 3D-Fernsehgeräte ziehen zu können. Die empirischen Daten aus den Fragebögen wurden mit Hilfe des Statistik-Programms SPSS (Version 19) ausgewertet. Die Forschungsergebnisse sind im vorliegenden Artikel zusammengefasst.

\section{Abstract}

According to estimates from several firm executives in consumer electronics as announced during the $» n$ ternationale Funkausstellung « (IFA) in Berlin in 2010 and 2011, the market for TVs with integrated 3D technology indicates very promising growth rates for the future. This relatively optimistic forecast was taken as an opportunity to initiate a field research project. Research activity targets to investigate the market potential for TV sets with 3D-technology. A total of 261 people from the Berlin-Brandenburg region were interviewed. Data allow deductions about market potentials for 3D TVs in the future. The empirical data were analyzed using the statistical program SPSS (version 19). Research outcomes are summarized in this paper.

\section{Einführung}

Initiiert von teilweise euphorischen Umsatzprognosen einiger Hersteller bzw. einschlägiger Marktforschungsinstitute während der Internationalen Funkausstellung 2010 und 2011 in Berlin, verfolgt die vorliegende Studie im Wesentlichen das Ziel, die sehr positiven Prognosen der Industrie für die kommenden Jahre in Bezug auf die Marktentwicklung von 3D-Fernsehgeräten zu hinterfragen. Aus diesem Grund wurde auf der Basis von Instrumenten der qualitativen Marktforschung eine Feldanalyse konzipiert. Mit Hilfe eines standardisierten Fragebogens sind unter anderem Marktpotenziale, Kaufbereitschaft und Preissensibilität von potentiellen Nachfragern untersucht worden. Im Zuge der empirischen Studie wurden insgesamt 261 Personen, vorwiegend im Raum Berlin-Brandenburg im 1. Halbjahr 2011 persönlich interviewt. Der Fragebogen umfasst sowohl offene als auch geschlossene Fragen. Im Zuge der Befragung wurde mit einer 7-wertigen Likert-Skala gearbeitet.

Im ersten Teil des Artikels werden Marketinginstrumente, insbesondere in ihrer Relevanz für die Konsumgüterindustrie, vorgestellt. Daran knüpft ein Überblick über die Entwicklung maßgebender Fernsehgerätetechnologien an. Im zweiten Teil folgt die empirische Datenauswertung aus der Umfrage in Hinblick auf Markenpräferenzen, eine Marktsegmentierung nach Kaufpreis und Geschlecht, bevorzugte Fernsehgerätetechnologien (z. B. LCD, Plasma) und Erfahrungen sowie Kaufbereitschaft. Abschließend werden auf Basis der gesammelten Erkenntnisse beeinflussende Faktoren für die 3D-Marktentwicklung sowie Empfehlungen für eine zukünftige Marktpositionierung herausgestellt. 


\section{Zum Begriff der Marketing- instrumente}

Das Spektrum der Marketinginstrumente umfasst die Produkt-, Preis-, Kommunikations- und Distributionspolitik (Kotler 2003: 16). Ein Produkt kann einen materiellen (Funktionalität eines TV-Gerätes) wie auch immateriellen Wert (Reputation) haben. Der integrierte Produktwert wird somit zum zentralen Aspekt für das Marketing, da eine Differenzierung zu den Wettbewerbern allein durch die Funktionalität nur schwer realisiert werden kann (Runia et al. 2007: 128; Meffert et al. 2006: 392; o.V. 2011a). Die ganzheitliche Umsetzung der zur Verfügung stehenden Marketinginstrumente hat einen entscheidenden Einfluss auf die erfolgreiche Umsetzung der avisierten Marketingziele.

Das Ziel der Produktpolitik liegt daher in der Entwicklung von funktional und qualitativ hochwertigen Gütern. Außerdem beinhaltet die Produktpolitik wesentliche Eigenschaften, ausgerichtet an den Bedürfnissen der Nachfrage, unter Berücksichtigung der durch den Wettbewerb gegebenen Randbedingungen sowie den wichtigen Faktoren Zeit und Kosten (Wöhe 2005: 485f.).

Für eine erfolgreiche Preispolitik sind neben den internen Faktoren (Kosten) auch die externen Rahmenbedingungen (Kunden, Marktpreise) relevante Größen (Runia et al. 2007: 181). In Bezug auf die Preispolitik werden im Wesentlichen zwei Ansätze unterschieden. Zum einen die klassische Preistheorie, die sich mit dem Absatzpreis auf vollkommenen Märkten beschäftigt. In einem vollkommenen Markt wird ein idealer Zustand angenommen, bei dem alle Anbieter und Nachfrager nach ökonomischen Grundsätzen handeln (Steuerer 2009: 6). Der zweite Ansatz, die praxisorientierte Preispolitik, beschäftigt sich mit der Optimierung des Absatzpreises auf unvollkommenen Märkten, welche in der Realität anzutreffen sind. Dabei kann der Preis entweder über den marktbasierten oder den kostenbasierten Preisansatz bestimmt werden. Im Zusammenhang mit dem marktorientierten Ansatz gibt es unter anderem die Penetrationsstrategie, bei der Neukunden durch relativ niedrige Preise gewonnen werden. Bei der Skimmingstrategie wird unmittelbar nach der Produkteinführung ein relativ hoher Preis verlangt, um die Amortisationsdauer der Produkte zu verkürzen (Meffert 2006: 553; Runia et al. 2007: 181f.). Die Skimmingstrategie wird vor allem für anspruchsvolle technologische Produkte, mit denen ein Innovationsvorsprung gene- riert werden soll, genutzt (Runia et al. 2007: 182). Ziel der Preispolitik ist daher unter anderem die strategische Gewinnmaximierung, um beispielsweise zukünftige Forschungs- und Entwicklungskosten finanzieren zu können (Wöhe 2005: 509f.).

In der Kommunikationspolitik werden neben der Unternehmenskultur auch neue Produktentwicklungen sowie die Preiswürdigkeit und die Bezugsquellen eines Produktes vermittelt (Wöhe 2005: 543). So wird in gesättigten Märkten neben der bekannten Unique Selling Proposition (USP), welche das Alleinstellungsmerkmal eines Unternehmens beschreibt, vor allem die Unique Advertising Proposition (UAP) innerhalb der Kommunikationspolitik genutzt. Dadurch soll die emotionale Alleinstellung des Produktes beim Kunden erzeugt und die werbliche Einzigartigkeit des Produktes betont werden (Runia et al. 2007: 223f.).

Die Distributionspolitik betrachtet sowohl die räumliche und zeitliche als auch die quantitative und qualitative Verfügbarkeit von Produkten. Unabhängig davon, ob es sich um eine direkte oder indirekte Distributionspolitik handelt, muss das Gut also am Ort der Nachfrage und unabhängig vom Zeitpunkt der Produktion verfügbar sein. Andererseits gilt es auch, bedarfsgerechte Mengen und gegebenenfalls Leistungsbündel sicherzustellen. Ebenso umfasst die Distributionspolitik die Planung von Transportmitteln und Transportwegen sowie Lagerstandorten und Lagerkapazitäten. Ziel ist hierbei die Optimierung des Absatzweges (Wöhe 2005: 564; Runia et al. 2007: 195).

Der Einsatz der beschriebenen Instrumente des Marketing-Mix ist sowohl untereinander als auch auf die jeweilige Marktsituation abzustimmen. Speziell die Konsumentengüterindustrie ist durch immer kürzer werdende Produktlebenszyklen gekennzeichnet. Dies trifft auch auf den Bereich der Fernsehgeräte zu, auf deren unterschiedliche Technologien im Folgenden kurz eingegangen wird.

\section{Technologie und Technologie- lebenszyklen}

$\mathrm{Zu}$ Beginn des 21. Jahrhunderts reduzierten die meisten TV-Gerätehersteller, wie beispielsweise Sony, Philips, Panasonic und Sharp, ihre Produktion von Röhrenfernsehgeräten (Hetzel 2006). Die charakteristische Kastenform, die Größe sowie das Gewicht waren die Hauptgründe für die Umorientierung der Nachfrager 
von konventionellen Röhrengeräten hin zu Flachbildfernsehern mit ansprechendem Design. Im Ergebnis wurden im Jahre 2006 in Europa mehr Flachbildgeräte als konventionelle Röhrenfernseher verkauft (Karnellos et al. 2006). Flachbildschirme funktionieren in der Regel auf Basis der Plasma- oder LCD-Technologie.

\section{LCD-Technologie}

Die Abkürzung LCD steht für Liquid Crystal Display (Flüssigkristallbildschirm) und die Funktionsweise dieser Technologie ist vergleichsweise kompliziert. Das Display besteht aus zwei dünnen Glasscheiben, deren Oberflächeninnenseiten mit einem speziellen polarisierenden Material überzogen sind (Elektrodenschicht). Zwischen den Glasscheiben befinden sich sogenannte Flüssigkristalle, die sich abhängig von der Stromzufuhr unterschiedlich verhalten: Sie können lichtdurchlässig oder lichtundurchlässig sein (o.V. 2011b).

Die Vorteile dieser Technologie liegen in dem geringen Stromverbrauch, dem flimmer- und verzerrungsfreien Bild, der relativ guten Helligkeit und einer maximalen Pixel-Anzahl. Vor allem im Monitorbereich sind LCD-Geräte meist auch wegen ihrer geringen Einbautiefe und ihres Gewichts besonders beliebt (o.V. 2011b).

LCD-Geräte haben aber auch einige technologisch bedingte Schwächen: LCD-Bildschirme beinhalten häufig schädliche Stoffe wie beispielsweise Cadmium, was einer gesonderten Entsorgung bedarf. Die Geräte sollten keinesfalls allzu extremen Temperaturen ausgesetzt werden, da sie vor allem bei Frost beschädigt werden können. Weiterhin zählen die eingeschränkte Farbwiedergabe (LCD-Fernseher stellen eher die Farbe Dunkelgrau als Schwarz dar) und die Abhängigkeit der Bildqualität vom Betrachtungswinkel zu den relevanten Nachteilen (o.V. 2011d; o.V. 2011e).

\section{Plasma-Technologie}

Der erste funktionsfähige Plasmabildschirm wurde bereits im Jahre 1964 von Donald L. Bitzer und H. Gene Slottow entwickelt. Jedoch erst über dreißig Jahre später, nämlich 1997, brachte das japanische Unternehmen Pioneer die ersten Plasmabildschirme in Fernsehgeräten auf den Markt (o.V. 2011f). Die Hersteller bewerben vor allem drei Eigenschaften der Plasmatechnologie: ein im Verhältnis zur LCD-Technologie geringerer Preis, die maximale Auflösung und ein besserer Kontrast (o.V. 2011g). Weitere Vorteile der Plasmatechnologie sind zum einen der sowohl vertikal als auch horizontal ausgeprägte Blickwinkel - ohne Farb- und
Kontrastbeeinträchtigungen - und die relativ kurze Reaktionszeit der einzelnen Bildzellen. Insgesamt sinkt jedoch der Marktanteil der Plasmatechnologie von Jahr zu Jahr. 2007 waren unter 4,4 Millionen verkauften Flachbildfernsehern in Deutschland lediglich 500.000 Plasmageräte. Einer der Gründe liegt darin, dass Plasma-TVs bis zu 30 Prozent mehr Energie als LCD-Fernseher benötigen (Masiero 2007). Alles in allem hat aber die Einführung der Plasma- und LCD-Fernsehgeräte vor über zehn Jahren den Markt massiv verändert. Plasma und LCD entwickelten sich zu dominierenden TVTechnologien und immer neue Modelle wurden sukzessive eingeführt.

\section{D-Technologie}

Nach den Aussagen führender Hersteller auf der Internationalen Funkausstellung 2010 und 2011 wird die 3D-Technologie in den kommenden Jahren den Markt bestimmen. Hier beruht die Funktionsweise auf dem Prinzip, dass der Mensch mit beiden Augen gleichzeitig verschiedene Bilder sieht. 3D-Effekte werden erreicht, indem das Bild in rote und grüne Bestandteile zerlegt wird, die dann in versetzten Positionen gezeigt werden. Um ein 3D-Bild für die Zuschauer zu erzeugen, tragen diese eine Brille, bei der eines der Gläser rot und das andere grün gefärbt ist. Damit wird erreicht, dass der gleiche Bildinhalt zweimal gleichzeitig gesehen wird, nur jeweils in unterschiedlichen Positionen durch das linke und rechte Auge. Auf diese Weise werden die roten und grünen Farben aufgrund der Schnelligkeit der Bildfolgen vom Auge gemischt wahrgenommen und somit ein dreidimensionales Bild im Kopf erzeugt (o.V. 2010a; o.V. 2011i; Pohl 2011).

Um ein dreidimensionales Bild im Gehirn entstehen zu lassen, existieren verschiedene Brillenvarianten. Bei einer sogenannten »Schutterbrille« handelt es sich um eine elektronisch gesteuerte Brille. Die Brillengläser werden (nach einer filmabhängigen Sequenz) abgedunkelt oder transparent geschaltet. Zusammen mit einem 3D-Film auf einem speziellen Fernsehgerät wird dann im Gehirn des Betrachters eine räumliche Wahrnehmung erzeugt. Der stereoskopisch aufgenommene Film, d. h. aus zwei Perspektiven aufgenommen, ist eine Voraussetzung für diese Technologie (Pohl 2011). »Polarisationsbrillen« unterscheidet man hauptsächlich an der Färbung der Brillengläser - transparent oder farbig. Bei beiden Ausprägungen beruht das Funktionsprinzip auf einer Filterung der einwirkenden Lichtwellen. Die transparenten Brillenfelder lassen nur Wellen aus 
einem bestimmten Winkel zum menschlichen Auge durchdringen. Dabei unterscheiden sich die Durchlasswinkel zwischen der linken und rechten Seite. Als Resultat erhält ein Auge eine andere Perspektive auf das betrachtete Objekt als das andere Auge. Das menschliche Gehirn erzeugt aus diesen beiden Perspektiven ein 3DBild. Bei den farbigen Polarisationsfolien spielt zwar die Ausrichtung der einfallenden Lichtwellen keine Rolle, jedoch aber die Wellenlänge. Die Wahrnehmung von Farben für das menschliche Auge ist abhängig von den unterschiedlichen Wellenlängen. So hat die Farbe Rot eine andere Wellenlänge als die Farbe Blau. Die farbigen Brillengläser erlauben daher nur einer bestimmten Farbe zum menschlichen Auge durchzudringen. Typische Farbenpaare sind rot/blau oder rot/grün (o.V. 2011j).

Darüber hinaus arbeiten Hersteller an Möglichkeiten, die das 3D-Fernsehen ohne zusätzliche Brille ermöglichen: Bei einer Variante besteht der Bildschirm aus Lentikularlinsen. Hier wird das Prinzip der holografischen Bilder genutzt, bei denen beim Wechsel der Perspektiven zum Bildschirm das Objekt im Raum erkannt wird. Bei einer anderen Variante wird das Bild über ein gewölbtes Display dargestellt, um einen 3D-Effekt ohne eine spezielle Brille zu erzeugen (Pohl 2011). Die Entwicklung der bereits heute verfügbaren, brillenlosen 3D-TV-Lösungen hin zur Marktreife wird daher in den nächsten Jahren zum neuralgischen Punkt für den Durchbruch des 3D-Fernsehens werden.

\section{Fernsehgerätehersteller im Überblick}

Im vergangenen Jahr zeichnete sich unter anderem durch den erfolgreichen Kinofilm Avatar ein steigendes Interesse in Zusammenhang mit der 3D-Technologie für den Heimgebrauch ab (Marth 2010). Gegenwärtig gibt es unter allen TV-Geräte Produzenten drei Hersteller, die sich durch eine innovative Technologie und eine entsprechende Qualität von ihren Wettbewerbern differenzieren: Samsung, Sharp und Panasonic (o.V. 2011k).

Mit mehr als 77 Prozent Marktanteil lagen die 3D-TVs von Samsung im Jahre 2010 in Deutschland an der Spitze (Schäfer 2010). 3D-Fernseher von Samsung weisen ein ansprechendes Design sowie relativ viele Modellvarianten auf (Staudt 2010a). Neben Premium-Produkten im höheren Preissegment entwickelt das Unternehmen aktuell weitere Produktserien für die mittlere Preisklasse, um zukünftig verstärkt Massenmärkte erschließen zu können und weitere Marktanteile zu gewinnen (Rößler 2011; Springer 2010).

Sharp präsentierte im Jahre 2010 den weltweit ersten 3D-LCD-Fernseher mit »Quattron Technologie«. $\mathrm{Zu}$ den ursprünglichen Grund-RGB-Farben (rot, grün, blau) ist noch eine vierte Farbe (gelb) hinzugekommen, was für eine verbesserte Farbwiedergabe sorgt (Staudt 2010a). Des Weiteren bietet Sharp nicht nur eines der besten 3D-Bilder bei Flachbildfernsehern, sondern bietet umweltschonende Produkteigenschaften, die im neuen AQUOS-Design integriert sind (Sharp Electronics 2010).

3D-Fernseher von Panasonic zeichnen sich durch eine gute Bewegungsschärfe, einem präzisen Kontrast sowie eine ausgezeichnete 3D-Effekt-Umsetzung aus (Staudt 2010a). Das Unternehmen vertreibt aktuell 3DGeräte mit einer Bildschirmdiagonale von 42 bis 65 Zoll (o.V.n.d.).

\section{Marktprognosen für 3D-TV- Fernsehgeräte}

Der Absatz wird momentan durch 2D-Flachbildschirme bestimmt. 2D-Flachbildschirme weisen in den meisten westeuropäischen Ländern heute eine Haushaltsdurchdringung von über 70 Prozent auf (GfK Retail and Technology 2011: 7). Einer Studie der Gesellschaft für Unterhaltungs- und Kommunikationselektronik (GfU) zur Folge wurden im Geschäftsjahr 2010 deutschlandweit gerade einmal 178.000 3D-Fernsehgeräte verkauft. Legt man die Verkaufszahlen für Fernsehgeräte insgesamt in Deutschland mit 9,7 Millionen Stück im Jahr 2010 als Vergleichsmaßstab an, so ergibt sich für 3D-Fernseher ein Marktanteil bei Neuverkäufen von lediglich rund 1,8 Prozent (Sawall 2011).

Nach Einschätzungen der GfU und dem Zentralverband Elektrotechnik- und Elektronikindustrie e.V. (ZVEI), ist das Marktpotenzial für 3D-Technologie in der Zukunft als außerordentlich positiv einzuschätzen. Gemäß einer im Vorfeld der Elektronikmesse IFA 2010 durchgeführten Studie wollen rund 41 Prozent der deutschen Konsumenten in den kommenden drei Jahren ein 3D-fähiges Fernsehgerät erwerben (Heller 2010). Dieser positiven Einschätzung folgend, prognostiziert auch die Consulting-Firma Goldmedia (Lehr n.d.) im Rahmen einer aktuellen Marktanalyse zum »3D-HomeEntertainment in Deutschland« einen rasanten Anstieg für 3D-TV-Geräte in den kommenden fünf Jahren. 
Angesichts sinkender Gerätepreise wird bis Ende 2011 ein Verkauf von 900.000 3D-fähigen Fernsehgeräten prognostiziert. In 2015 sollen nach Goldmedia dann bereits rund 8,4 Millionen Haushalte in Deutschland, also knapp ein Fünftel aller Haushalte, mit einem 3DFernseher ausgestattet sein (Display Search 2010).

Qualitativ zufriedenstellende Endgeräte sind für eine Marktdurchdringung der 3D-Technologie erfolgsentscheidend. Gleichzeitig muss auch ein korrespondierendes Programmangebot am Markt verfügbar sein. Hier liegt zudem eine weitere Herausforderung, denn aufgrund der zusätzlichen Kosten für spezielle 3D-Kameras und neue Regiekonzepte sind 3D-Produktionen immer noch deutlich teurer als zweidimensionale TVProduktionen. Daher gibt es aktuell nur sehr wenige dreidimensionale TV-Inhalte im deutschen Fernsehen (Lehr n.d.). Zudem konzentrieren sich die Fernsehsender derzeit auf die einwandfreie Bereitstellung von HDTV (hochauflösendes Fernsehen) sowie den Ausbau eines entsprechenden HDTV-Programmangebots (Deutsche TV-Plattform 2010). Andererseits arbeiten internationale Gremien an einer Standardisierung für einzelne Spezifikationen der dreidimensionalen Fernsehübertragung, was der Verbreitung von 3D-TV neuen Aufschwung geben würde. Auch das Angebot für 3D-Kinoproduktionen auf Blu-ray-Datenträgern dürfte sich in den kommenden Jahren möglicherweise positiv entwickeln (Fachverband Consumer Electronics 2010).

Für die Zukunft ist folgendes Szenario vorstellbar: TVGeräteproduzenten, welche die 3D-Funktionalität von Fernsehgeräten neben der Internetfähigkeit als wichtigstes Zusatzmerkmal identifiziert haben, werden in den kommenden Jahren verstärkt Geräte im mittleren Preissegment anbieten (GfU 2010: 1). Bedingt durch zunehmenden Preisdruck sowie eine größere Vielfalt an 3D-Inhalten wird sich das Konsumenteninteresse an 3D-fähigen TV-Geräten, Experteneinschätzungen zufolge, entsprechend positiv entwickeln. Für 2015 erwartet man daher einen weltweiten Absatz von 159,2 Millionen 3D-Fernsehern. Bei diesem Szenario muss berücksichtigt werden, dass die TV-Gerätehersteller 3D grundsätzlich zu einer festen Funktion (Zusatznutzen) in ihren Geräten machen und damit der Marktanteil an reinen 2D-Fernsehern zwangsweise reduziert wird (Karthaus 2011). Im nachfolgenden Abschnitt wird im Ergebnis einer empirischen Feldbefragung im Raum Berlin-Brandenburg das Meinungsbild zu Markenbild, Kaufpreis, TV-Technologie Präferenzen, Erfahrungen mit 3D-Technik und das potentielle Interesse für 3D zu- sammengefasst. Hintergrund ist die Fragestellung, ob die relativ optimistischen Vorhersagen der Industrie in Bezug auf 3D derzeit im Markt reflektiert werden oder nicht.

\section{Datenauswertung}

Über zwei Drittel der befragten Personen haben beim Kauf eines neuen Fernsehgerätes klare Präferenzen bezüglich der Marke. Auffällig ist hier die Beliebtheit des Herstellers Sony: ca. 40 Prozent bevorzugen ein solches Gerät. Von den übrigen Personen mit einer klaren Markenpräferenz wählten rund ein Viertel die Marke Samsung. Philips erreicht einen Wert von ca. 14 Prozent. Darüber hinaus, allerdings mit Abstand, finden sich Panasonic, LG Electronics und Loewe in der Kategorie der beliebtesten Marken wieder (vgl. Abbildung 1).

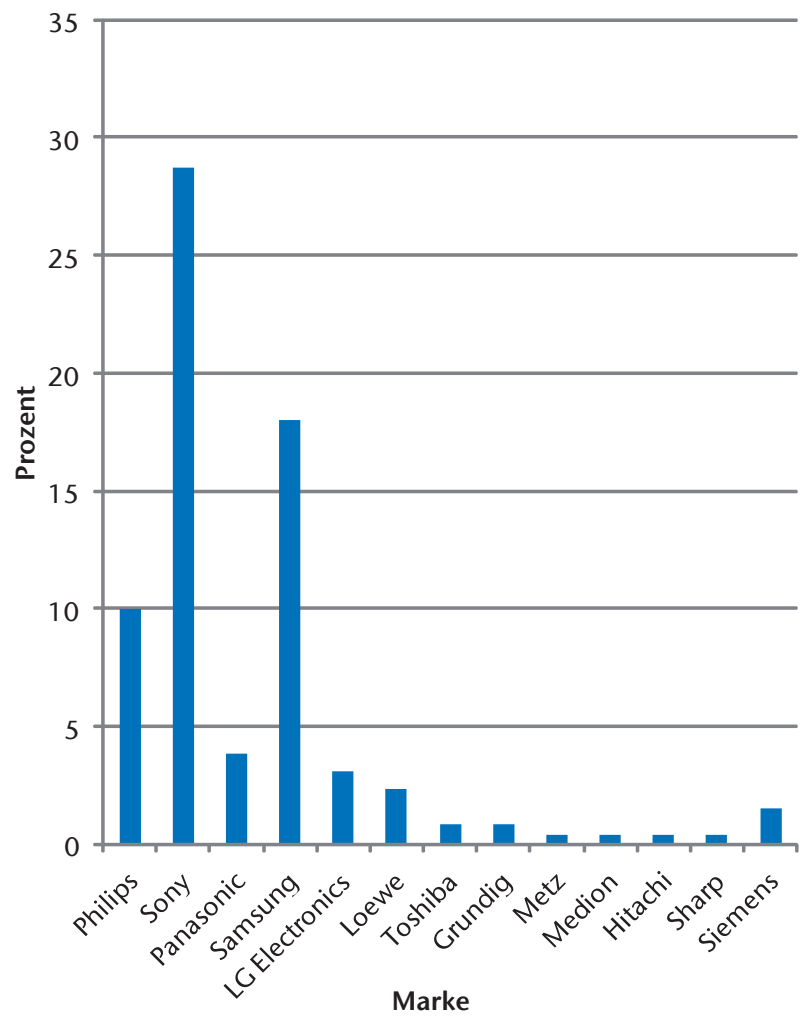

Abb. 1: Bevorzugte Marken in der Stichprobe (261 Fragebögen - Stand Juni 2011)

Die Auswertung des empirischen Datenmaterials hat ergeben, dass die Mehrheit der Befragten zwischen 600 Euro und 1.000 Euro für einen neuen Fernseher ausgeben würde (rund 55 Prozent). Interessante Ergebnisse liefert eine Gegenüberstellung der Antworten nach 
geschlechterspezifischen Aspekten. Es ist deutlich zu erkennen, dass männliche Befragte offensichtlich eher dazu bereit sind, mehr Geld für ein neues Fernsehgerät auszugeben als die weiblichen Konsumenten. Die männlichen Befragten habe die Preisspanne »bis 1.000 Euro« am häufigsten genannt. Die Mehrzahl der weiblichen Teilnehmerinnen in der Studie würde nur »bis 600 Euro« in ein neues Gerät investieren. In der Tabelle 1 sind die Ergebnisse zusammenfassend dargestellt.

\begin{tabular}{|c|c|c|c|c|}
\hline & & weiblich & männlich & Gesamt \\
\hline maxi- & keine Präferenz & 11 & 8 & 19 \\
\hline maler & bis 200 Euro & 6 & 6 & 12 \\
\hline preis & bis 400 Euro & 12 & 2 & 14 \\
\hline & bis 600 Euro & 28 & 20 & 48 \\
\hline & bis 800 Euro & 18 & 25 & 43 \\
\hline & bis 1000 Euro & 13 & 34 & 47 \\
\hline & bis 1200 Euro & 10 & 13 & 23 \\
\hline & bis 1500 Euro & 11 & 13 & 24 \\
\hline & bis 2000 Euro & 4 & 8 & 12 \\
\hline & über 2000 Euro & 4 & 8 & 12 \\
\hline Gesan & & 117 & 137 & 254 \\
\hline
\end{tabular}

Tab. 1: Maximaler Kaufpreis (männlich vs. weiblich)

In Bezug auf die präferierten Fernsehtechnologien dominieren, mit rund 60 Prozent, die LCD-Fernseher. Mit großem Abstand folgen Plasmafernseher (rund 16 Prozent). Acht Prozent der Befragten haben keine Prä-

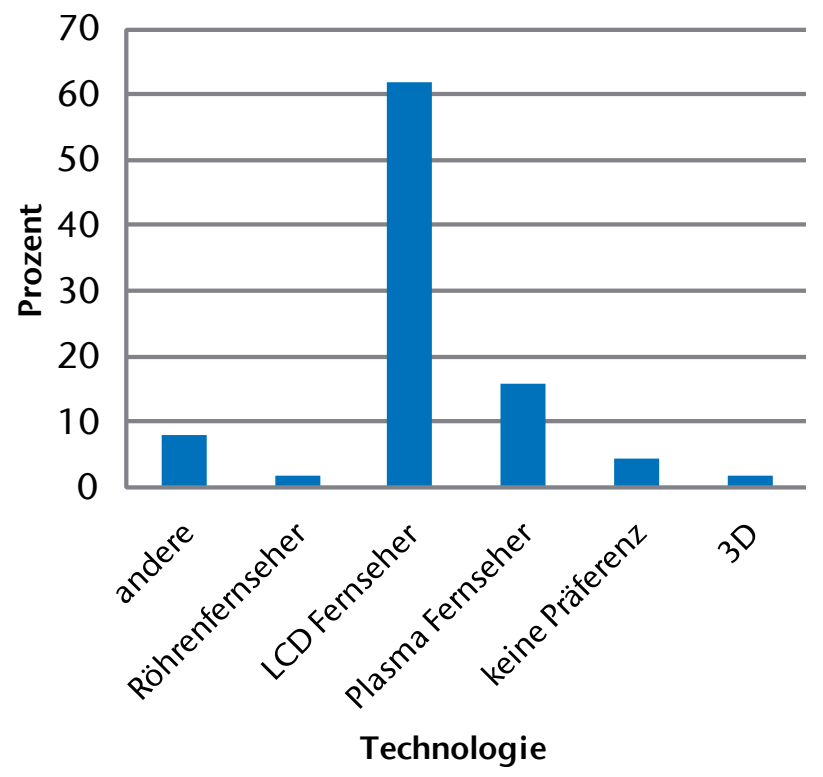

Abb. 2: Präferenzen in Bezug auf TV-Technologien ferenz bezüglich der derzeit am Markt angebotenen Technologien. Besonders hervorzuheben ist das Ergebnis aber im Hinblick auf die 3D-Technologie. Lediglich rund 2 Prozent der Befragten gaben an, einen 3D-Fernseher gegenüber einem konventionellen 2D-Fernsehgerät zu bevorzugen (vgl. Abbildung 2).

Bezogen auf die Frage, ob und welche Erfahrungen bereits mit 3D-Fernsehern gemacht wurden, zeigt die Analyse der verwertbaren 164 Antworten deutlich, dass die Mehrheit der Befragten (123 Personen) zum Zeitpunkt der Datenerhebung noch nicht mit der neuen Technologie in Berührung kamen. Lediglich rund neun Prozent (23 Personen) gaben bei dieser Frage ein positives Urteil ab, während rund sieben Prozent (18 Befragte) negative Erfahrungen mit der 3D-Technologie gemacht haben. Der relativ hohe Anteil negativer Erfahrungen sollte für die etablierten 3D-Fernsehgerätehersteller ein Warnsignal sein. Es ist daraus zu schließen, dass noch erhebliche Anstrengungen in der Produktweiterentwicklung notwendig sind (vgl. Tabelle 2).

\begin{tabular}{|c|c|c|c|c|c|}
\hline & & Häufigkeit & Prozent & $\begin{array}{c}\text { Gültige } \\
\text { Prozente }\end{array}$ & $\begin{array}{r}\text { Kumulierte } \\
\text { Prozente }\end{array}$ \\
\hline \multirow[t]{4}{*}{ Gültig } & positiv & 23 & 8,8 & 14,0 & 14,0 \\
\hline & negativ & 18 & 6,9 & 11,0 & 25,0 \\
\hline & $\begin{array}{l}\text { keine Erfah- } \\
\text { rungen }\end{array}$ & 123 & 47,1 & 75,0 & 100,0 \\
\hline & Gesamt & 164 & 62,8 & 100,0 & \\
\hline Fehlend & $\begin{array}{l}\text { keine } \\
\text { Angabe }\end{array}$ & 97 & 37,2 & & \\
\hline Gesamt & & 261 & 100,0 & & \\
\hline
\end{tabular}

Tab. 2: Erfahrungen mit 3D-Fernsehgerätetechnologie

Für rund drei Viertel der Befragten kommt derzeit ein Wechsel von einem konventionellen 2D- zu einem 3Dfähigen Gerät nicht in Frage. Lediglich rund 25 Prozent der Befragten wären bereit, ihr momentanes Fernsehgerät gegen einen 3D-Fernseher zu tauschen (vgl. Tabelle $3)$.

\begin{tabular}{|l|l|r|r|}
\hline \multirow{2}{*}{ Gültig } & \multicolumn{2}{|c|}{ Häufigkeit } & Prozent \\
\cline { 2 - 3 } & ja & 40 & 15,3 \\
\hline & nein & 124 & 47,5 \\
\hline & Sub-Total & 164 & 62,8 \\
\hline & keine Angabe & 97 & 37,2 \\
\hline Total & & $\mathbf{2 6 1}$ & $\mathbf{1 0 0 , 0}$ \\
\hline
\end{tabular}

Tab. 3: Ersatz von 2D- durch 3D-Fernseher 
Von den insgesamt 119 verwertbaren Antworten aus der Stichprobe gaben 43 Befragte an, einen Mehrpreis von bis zu zehn Prozent für ein 3D-Gerät im Vergleich zu einem konventionellen 2D-TV akzeptieren zu wollen. Rund die Hälfte der befragten Personen (51 Teilnehmer) würde bis zu 20 Prozent höhere Anschaffungskosten für einen 3D-Fernseher akzeptieren. Ein signifikanter Rückgang der Kaufbereitschaft wird bei Mehrkosten von bis zu 30 Prozent oder darüber hinaus deutlich. Diese Aussagen verdeutlichen, dass die Konsumenten mit Zurückhaltung auf die 3D-Technologie reagieren. Somit kann die vorliegende Studie zumindest für den Raum Berlin-Brandenburg die eher optimistischen Einschätzungen der Industrie in Bezug auf die zukünftige Marktentwicklung der 3D-Technologie nicht bestätigen.

\section{Fazit}

Zusammenfassend kann konstatiert werden, dass die Mehrheit der Befragten, zumindest in dieser Stichprobe, noch wenig Interesse für die 3D-Fernsehtechnologie entwickelt hat. Ein Kriterium für die zurückhaltende Kaufbereitschaft ist nach den Ergebnissen der Studie die Brille. Um den Marktdurchbruch von 3D zu erwirken, scheint es notwendig, dass die Industrie 3D-Fernsehen ohne eine subjektiv oder real (z. B. Brillenträger) als störend empfundene Brille ermöglicht. Außerdem müsste sich die Film- und Fernsehindustrie verstärkt dahingehend ausrichten, das Angebot an 3D-Inhalten für den privaten Haushalt auszuweiten.

Die Studie liefert darüber hinaus Erkenntnisse, dass die Mehrheit der Befragten Zusatzkosten bis maximal 20 Prozent des Kaufpreises für ein 3D-Gerät im Vergleich zu einem konventionellen 2D-Fernseher akzeptieren würde. Diese psychologische Grenze gilt es für die Hersteller im Rahmen ihrer Preispolitik zu berücksichtigen. Alarmierend ist aber der relativ hohe Anteil derjenigen mit negativen Erfahrungen bezüglich der 3D-Technologie. Wenn die Faktoren »3D-Brille«, Ausweitung des dreidimensionalen Filmangebotes, Produktperformance und Preis von den TV-Geräteherstellern nicht in entsprechendem Maße berücksichtigt werden, kommt die Studie zu dem Schluss, dass die vielversprechenden Marktprognosen der Industrie in Bezug auf die 3D-Technologie für die kommenden Jahre nicht realistisch sind.

\section{Literatur}

Deutsche TV-Plattform (n.d.): Arbeitsgruppen: 3D-HD-TV, http:// www.tv-plattform.de/de/arbeitsgruppen/3d-hd-tv.html, Zugriff: 12.05.2011.

Deutsche TV-Plattform (2010): Presseinformation: Top-Thema dreidimensionales Fernsehen beim 19. Symposium der Deutschen TV-Plattform in Berlin, http://www.tv-plattform.de/de/oeffentlichkeitsarbeit/pressemitteilungen.html, Zugriff: 12.05.2011.

Display Search (2010): TV market analysis. IFA Conference Berlin, September 2010.

Fachverband Consumer Electronics (2010): 3D-Spaß unter dem Weihnachtsbaum, http://www.zvei.org/fachverbaende/consumer_electronics/nachrichten/archiv_2010/newsdetail/3d_spass_ unter_dem_weihnachtsbaum/, Zugriff: 12.05.2011.

Gesellschaft für Unterhaltungs- und Kommunikationselektronik (GfU) (2010): IFA 2010: Trends der Consumer Electronics, http:// www.gfu.de/home/consumer/technologien.xhtml, Zugriff: 07.05.2011.

GfK Retail and Technology (2011): Pressemitteilung. Westeuropäische Märkte für technische Gebrauchsgüter im vierten Quartal 2010 stabil. Ergebnisse des GfK TEMAX ${ }^{\oplus}$ Westeuropa für das vierte Quartal und das Gesamtjahr 2010, http://www.gfk.com/group/ press_information/press_releases/007437/index.de.html, Zugriff: 12.05.2011.

Heller, H. (2010): Elektronik Praxis. 3D Fernseher stoßen auf hohes Interesse, http://www.elektronikpraxis.vogel.de/marktzahlen/ articles/276316/, Zugriff: 06.05.2011.

Hetzel, H. (2006): LG Philips beendet Ära des Röhren-Fernseher in Europa, http://www.welt.de/print-welt/article197245/LG_Philips_beendet_Aera_der_Roehren_Fernseher_in_Europa.html, Zugriff: 26.05.2011

Karnellos, M., Greif, B. (2006): LCD-TVs verkaufen sich besser als Röhrenfernseher, http://www.zdnet.de/news/wirtschaft_unternehmen_business_lcd_tvs_verkaufen_sich_besser_als_roehrenfernseher_story-39001020-39149346-1.htm, Zugriff: 23.05.2011.

Karthaus, O. (2011): Marktforscher: 3D-TV-Verkäufe legen 2011 um 463 Prozent zu, http://www.hddaily.de/2011/05/10/marktforscher-3d-tv-verkaufe-legen-2011-um-463-prozent-zu-29021.html, Zugriff: 12.05.2011.

Kotler, P. (2003): Marketingmanagement. Prentice Hall International, New Jersey.

Lehr, S. (n.d.): Home-Entertainment wird dreidimensional: Nach Kino und Gaming erobert 3D 2011 auch die mobilen Displays, 3D-TVInhalte bleiben hingegen spärlich, http://www.goldmedia.com/ aktuelles/trendmonitor-2011/3d-home.html, Zugriff: 12.05.2011.

Marth, M. (2010): Die Trends der Funkausstellung, http://www.focus. de/digital/multimedia/ifa-2010/tid-19632/ifa-2010-die-trendsder-funkausstellung_aid_545669.html, Zugriff: 05.06.2011.

Masiero, M. (2007): Plasma oder LCD: Einkaufsberater Flachbild-Fernseher, http://www.netzwelt.de/news/76803-plasma-lcd-einkaufsberater-flachbild-fernseher.html, Zugriff: 20.04.2011.

Meffert, H., Bruhn, M. (2006): Dienstleistungsmarketing: Grundlagen - Konzepte - Methoden ( 5. Auflage). Gabler Verlag, Wiesbaden.

Niezijewski, A. (2011): Der Röhrenfernseher, http://www.das-heimkino.com/html/rohrenfernseher.html, Zugriff: 23.04.2011.

o.V. (2010a): 3D Fernsehen, http://www.hifi-regler.de/panasonic/3dfernsehen.php?SID=2603f9ee64cd8516dfce95c28634c80c, Zugriff: 21.05.2011 
o.V. (2010b): 2010 Best of CES winner is Panasonic‘s 3D plasma TV, http://ces.cnet.com/8301-31045_1-10431350-269.html\%20 2011-05-18\%2017:53, Zugriff: 18.05.2011.

o.V. (2011a): Der Begriff des Produktes, http://www.teialehrbuch.de/ Kostenlose-Kurse/Marketing/15240-Der-Begriff-des-Produktes. html, Zugriff: 21.05.2011.

o.V. (2011b): Was ist eigentlich LCD? http://www.flimmerkisten.de/ was-ist-eigentlich-lcd-lexikon-319.html, Zugriff: 26.04.2011.

o.V. (2011d): LCD-Fernseher Vorteile-Nachteile, http://www.sed-fernseher.eu/lcd-fernseher-vorteile-nachteile, Zugriff: 20.04.2011.

o.V. (2011e): Vorteile Nachteile Plasma, http://www.hifi.ch/vorteilenachteile-plasma-lcd.html, Zugriff: 20.04.2011.

o.V. (2011f): Plasma Fernseher Geschichte, http://www.sed-fernseher. eu/plasma-fernseher-geschichte, Zugriff: 24.04.2011.

o.V. (2011g): Plasmabildschirm, http://www.hardware-aktuell.com/ lexikon/Plasmabildschirm, Zugriff: 20.04.2011.

o.V. (2011i): Wie funktioniert 3D eigentlich? http://www.3dtv-infos de/index.php?id=16, Zugriff: 21.04.2011

o.V. (2011j): 3D-Brillen, http://www.3d-brillen.de/3d-brillen/, Zugriff: 21.04.2011.

o.V. (2011k): 3D gewinnt 2011 mächtig an Fahrt, http://www.mediabiz.de/video/news/3d-gewinnt-2011-maechtig-an-fahrt/304849, Zugriff: 10.05.2011.

o.V. (n.d.): 2011 - Welchen 3D Fernseher kaufen, http://pagewizz. com/2011-welchen-3d-fernseher-kaufen/, Zugriff: 18.05.2011.

Pohl, T. (2011): 3D-Fernseher, http://www.3dfernseher.tv/, Zugriff: 10.05.2011.

Rößler, M. (2011): 3D-Kauftipps: Für TV-Sparfüchse und TechnikFreaks, http://www.chip.de/artikel/3D-Fernseher-TV-Ratgeber-und-Kaufberatung-fuer-3D-6_45828841.html, Zugriff: 10.05.2011

Runia, P., Wahl, F., Geyer, O., Thewißen, C. (2007): Marketing: Eine Prozess- und Praxisorientierte Einführung (2. Auflage). Oldenbourg Verlag, München

Sawall, A. (2011): Erste 3D-TV-Verkaufszahlen für Deutschland veröffentlicht, http://www.golem.de/1102/81187.html, Zugriff: 12.05.2011.

Schäfer, F. (2010): Samsung verteidigt Spitzenposition, TV-Hersteller: Samsung ist seit 5 Jahren »on Top«, http://www.tvfacts.de/ artikel/4062-tv-hersteller-samsung-seit-5-jahren-on-top.html, Zugriff: 18.05.2011.

Schonscheck, O. (2006): Der Fernseher: Die Geschichte eines treuen Freundes, http://news.idealo.de/news/1515-der-fernseher-diegeschichte-eines-treuen-freundes/, Zugriff: 05.05.2011.

Sharp Electronics (2010): Sharp präsentiert weltweit ersten 3D-TV mit Quattron Technologie, http://www.sharp.de/cps/rde/xchg/de/ hs.xsl/-/html/25538.htm, Zugriff: 18.05.2011.

Springer, O. (2010): Top 10: Die aktuell 10 besten 3D Fernseher 2010, http://news.preisgenau.de/top-10-die-aktuell-10-besten-3d-fernseher-2010-6487.html, Zugriff: 18.05.2011.

Staudt, M. (2010a): 3D Fernseher Vergleich \& Test: Sony, Samsung etc. die 3DTV Liste, http://www.3d-entertainment.net/3d-fernsehervergleich-test-sony-samsung-etc-die-3d-tv-liste/10928, Zugriff: 18.05.2011.

Staudt, M. (2010b): 3D-Fernseher: Verkaufszahlen für Europa - 25.000 3D-TVs verkauft, http://www.3d-entertainment.net/3d-fernseher-verkaufszahlen-europa/10801, Zugriff: 10.05.2011.

Stepanek, M. J. (2010): 3D-fähige TV-Geräte sind Ladenhüter - Mittleres Preissegment vernachlässigt, http://www.pressemitteilungenonline.de/index.php/3d-faehige-tv-geraete-sind-ladenhuetermittleres-preissegment-vernachlaessigt, Zugriff: 10.05.2011.
Steuerer, M. (2009): Die risikogerechte Prämie in der Kfz-Haftpflichtversicherung: Probleme der Preisgestaltung auf Märkten mit asymmetrischen Informationen. GRIN Verlag, München.

Wöhe, G. (2005): Einführung in die allgemeine Betriebswirtschaftslehre (22. Auflage). Verlag Franz Vahlen, München.

\section{Autoren}

Prof. Dr. Mario Glowik

Fachbereich Betriebswirtschaft/Wirtschaftsinformatik

Technische Hochschule Wildau [FH]

mario.glowik@th-wildau.de

Jenny Baumann

Sarah Bruhs

Yelena Bryantseva

Ekaterina Chmyreva

Marie-Christin Ernst

Anne Frischbier

Katharina Fritsch

Lisa Lippert

Bo Ma

Thuy Nguyen

Nathalie Sroka 